\title{
ON THE COMPACTNESS FOR TWO DIMENSIONAL SCALAR CONSERVATION LAW WITH DISCONTINUOUS FLUX*
}

\author{
JELENA ALEKSIĆ ${ }^{\dagger}$ AND DARKO MITROVIC ${ }^{\ddagger}$
}

Abstract. We prove that a family of solutions to a Cauchy problem for a two dimensional scalar conservation law with a discontinuous smoothed flux and the vanishing viscosity is strongly $L_{\text {loc }}^{1}$ - precompact under a new genuine nonlinearity condition, weaker than in previous works on the subject.

Key words. Scalar conservation law, discontinuous flux, vanishing viscosity, regularized flux, strong $L_{\text {loc }}^{1}$-precompactness, $H$-measures.

AMS subject classifications. 35L65, 35L80.

\section{Introduction}

We consider two dimensional variant of the following Cauchy problem for a scalar conservation law

$$
\begin{aligned}
u_{t}+\operatorname{div}_{x} f(x, u) & =0, \\
u(0, x) & =u_{0}(x),
\end{aligned}
$$

where $u=u(t, x), x \in \mathbf{R}^{d}, t \in \mathbf{R}^{+}$, and $f=\left(f_{1}, \ldots, f_{d}\right): \mathbf{R}^{d+1} \rightarrow \mathbf{R}^{d}, d \in \mathbf{N}$. For the initial data $u_{0}$ we assume that

$$
u_{0} \in\left(B V \cap L^{\infty}\right)\left(\mathbf{R}^{d}\right), \quad a \leq u_{0}(x) \leq b, \quad x \in \mathbf{R}^{d},
$$

where $a, b \in \mathbf{R}$ are constants. The flux function $f=\left(f_{1}, \ldots, f_{d}\right)$ has the following properties:

$$
\begin{aligned}
& f_{i}(\cdot, \lambda) \in\left(B V \cap L^{\infty}\right)\left(\mathbf{R}^{d}\right) \text { for every } \lambda \in \mathbf{R}, \\
& f_{i}(x, \cdot) \in \operatorname{Lip}(\mathbf{R}) \text { for every } x \in \mathbf{R}^{d} \\
& \max _{a \leq u \leq b}\left|f_{i}(\cdot, u)\right|_{B V\left(\mathbf{R}^{d}\right)}<\infty, \max _{a \leq u \leq b}\left|f_{i}(\cdot, u)\right| \in L_{\text {loc }}^{q}\left(\mathbf{R}^{d}\right) \text { for some } q>2, \\
& 0=f_{i}(x, b)=f_{i}(x, a) \quad \text { for every } x \in \mathbf{R}^{d} \text { and } i=1, \ldots, d,
\end{aligned}
$$

where $\operatorname{Lip}(\mathbf{R})$ denotes a space of Lipschitz continuous functions.

In recent years, problems of this kind received lots of attention since they model many physical phenomena. As examples of special importance, we emphasize applications in flow in porous media, sedimentation processes, traffic flow, radar shapefrom-shading problems, blood flow, and gas flow in a variable duct.

If $f_{i}, i=1, \ldots, d$, are smooth functions, then the existence and uniqueness of an entropy solution is provided by the well known method of doubling of variables due

*Received: July 13, 2009; accepted (in revised version): October 12, 2009. Communicated by Eitan Tadmor.

The work of the authors is supported in part by the the local government of municipality Budva and DAAD project "Center of Excellence for Applications of Mathematics" within the framework of the DAAD programme "Academic reconstruction of South-East Europe".

${ }^{\dagger}$ Department of Mathematics and Informatics, University of Novi Sad, Trg D. Obradovića 4, 21000 Novi Sad, Serbia (jelena.aleksic@dmi.uns.ac.rs).

$\ddagger$ Faculty of Mathematics and Natural Sciences, University of Montenegro, Cetinjski put bb, 81000 Podgorica, Montenegro (matematika@t-com.me). 
to Kružkov [16], or by using the measure valued concept by DiPerna [8]. It is well known, cf. [6, 16], that for the Lipschitz continuous flux, the family of solutions to the vanishing viscosity regularization of (1.1) (see (1.8)-(1.9) below) converges to a weak solution of (1.1) in the strong $L_{\text {loc }}^{1}\left(\mathbf{R}^{+} \times \mathbf{R}^{d}\right)$ topology. However, if the flux is discontinuous with respect to $x \in \mathbf{R}^{d}$, we can not apply a classical approach.

The existence of a weak solution for the two dimensional variant of (1.1) was settled in [12] for a flux in a slightly less general form satisfying the following genuine nonlinearity condition. Let $S^{2} \subset \mathbf{R}^{3}$ denotes the unit sphere. We say that the flux $\left(f_{1}, f_{2}\right)$ satisfies a genuine nonlinearity condition if

$$
\text { for almost every } \begin{aligned}
x & \in \mathbf{R}^{2} \text { and every } \xi=\left(\xi_{0}, \xi_{1}, \xi_{2}\right) \in S^{2} \text { the mapping } \\
\lambda & \mapsto \xi_{0} \lambda+f_{1}(x, \lambda) \xi_{1}+f_{2}(x, \lambda) \xi_{2}
\end{aligned}
$$

is not constant in $\lambda$ on any nontrivial interval.

The existence was obtained as a consequence of the strong $L_{\text {loc }}^{1}\left(\mathbf{R}^{+} \times \mathbf{R}^{2}\right)$ precompactness of a family of solutions to (1.8). The proof is based on a two dimensional variant [19] of the method of compensated compactness [20].

More precisely, the following regularization of problem (1.1) with $d=2$ was considered (here and in the following $\Delta$ stands for the Laplacian $\Delta u=\sum_{i=1}^{d} u_{x_{i} x_{i}}$ and $\left.x=\left(x_{1}, \ldots, x_{d}\right) \in \mathbf{R}^{d}\right):$

$$
\begin{aligned}
u_{t}^{\varepsilon, \delta}+\operatorname{div} f^{\delta}\left(x, u^{\varepsilon, \delta}\right) & =\varepsilon \Delta u^{\varepsilon, \delta} \\
\left.u^{\varepsilon, \delta}\right|_{t=0} & =u_{0}^{\delta},
\end{aligned}
$$

where the approximations $f^{\delta}=\left(f_{1}^{\delta}, \ldots, f_{d}^{\delta}\right)$ and $u_{0}^{\delta}$ are constructed in the following manner. Let $\omega: \mathbf{R} \rightarrow \mathbf{R}$ be an arbitrary smooth function such that $\omega(\xi)=0$ for $|\xi| \geq 1$, and $\int_{\mathbf{R}} \omega(\xi) d \xi=1$. We define for every $i=1, \ldots, d$ (we consider $\lambda$ as a parameter below)

$$
f_{i}^{\delta}(x, \lambda)=\frac{1}{\delta^{d}} \Pi_{i=1}^{d} \omega\left(x_{i} / \delta\right) \star f_{i}(x, \lambda),
$$

and

$$
u_{0}^{\delta}(x)=\frac{1}{\delta^{d}} \Pi_{i=1}^{d} \omega\left(x_{i} / \delta\right) \star\left(u_{0} \chi_{\delta}\right)(x),
$$

where $\chi_{\delta}(x)=1$ for $x \in B(0,1 / \delta) \subset \mathbf{R}^{d}$ and zero otherwise. Here, $\star$ stands for the convolution operator.

The case of an arbitrary dimension space was completed by Panov [18], using another method of Tartar - $H$-measures [21] (introduced independently by Gerard [10] who named them microlocal defect measures). Similar to [12], in [18] it was proved that a family of solutions to equation (1.1) with the regularized flux is strongly $L_{\text {loc }}^{1}$ precompact under a multidimensional variant of genuine nonlinearity condition (1.7).

We stress that in the one dimensional case, one does not need any nonlinearity condition in order to prove the existence of a weak solution to a scalar conservation law with a flux discontinuous in the space variable. More precisely, using the compensated compactness argument [14] it is not difficult to prove that a family of entropy admissible solutions $[12,18]$ of the one dimensional variant of (1.8) converges weakly along a subsequence to a solution of the one dimensional variant of (1.1). 
However, we can not state anything about strong $L_{\text {loc }}^{1}$-precompactness of the family $\left(u^{\varepsilon, \delta}\right)_{\varepsilon, \delta}$ (see [13, Remark 2.3]), which is of essential importance since a strong $L_{\text {loc }}^{1}$-limit along a subsequence of $\left(u^{\varepsilon, \delta}\right)_{\varepsilon, \delta}$ satisfies admissibility conditions (see [14, Definition 1.2]). On the other hand, such conditions provide a stability of solutions for problems of type (1.1) (see e.g. [5, 14]).

In this paper, we shall prove that under a relaxed genuine nonlinearity condition (see (2.1) below), a family of solutions to (1.8) with $d=2$ is strongly precompact in $L_{\text {loc }}^{1}\left(\mathbf{R}^{+} \times \mathbf{R}^{2}\right)$. This will provide the existence of a weak solution to (1.1) when the flux $f=\left(f_{1}, f_{2}\right)$ is not necessarily genuinely nonlinear, which is actually the main contribution of the paper. As a consequence, in two physically relevant one dimensional situations of the problem, we are able to prove strong $L_{\text {loc }}^{1}$-precompactness of the family $\left(u^{\varepsilon, \delta}\right)_{\varepsilon, \delta}$ merely by assuming that the initial data belongs to the BV-class (which actually proves the existence of an entropy admissible weak solution to the one-dimensional variant of (1.1) without any additional assumptions on the flux). For the latter, see section 3.

In order to get the result, we shall use estimates derived in [12] and the following theorem.

TheOREm 1.1 ([18], Corollary 2). Let $\Omega \subset \mathbf{R}^{n}$ be an open set. Assume that the vector $\phi(x, u) \in\left(C\left(\mathbf{R}_{u} ; B V(\Omega)\right)\right)^{n}$ is genuinely nonlinear, i.e., for a.e. $x \in \Omega$ and for all $\xi \in \mathbf{R}^{n}, \xi \neq 0$, the map $(a, b) \ni u \mapsto(\xi, \phi(x, u)) \neq$ constant on any nontrivial interval.

Then, each bounded sequence $\left(u_{k}(x)\right)_{k} \in L^{\infty}(\Omega), a \leq u_{k}(x) \leq b$, satisfying for the Heaviside function $H$,

$$
\operatorname{div}_{x}\left[H\left(u_{k}(x)-p\right)\left(\phi\left(x, u_{k}(x)\right)-\phi(x, p)\right)\right] \text { is precompact in } W_{\text {loc }}^{-1,2}(\Omega),
$$

contains a subsequence that is convergent in $L_{\mathrm{loc}}^{1}(\Omega)$.

The key point of our procedure is the fact that for the family of solutions $\left(u^{\varepsilon, \delta}\right)_{\varepsilon, \delta}$ of (1.8), (1.9), $\left\|u_{t}^{\varepsilon, \delta}(t, \cdot)\right\|_{L^{1}\left(\mathbf{R}^{2}\right)}$ is uniformly bounded with respect to $\epsilon, \delta$ for every $t>0$. Therefore, we can replace $u_{t}^{\varepsilon, \delta}$ by a function $\partial_{t} h\left(x, u^{\varepsilon, \delta}\right)$ (actually, $u_{t}^{\varepsilon, \delta}$ will end up on the right hand side) without affecting the precompactness framework. This means that we can replace $\xi_{0} \lambda$ from $(1.7)$ by $\xi_{0} h(x, \lambda)$ where $h$ is chosen so that conditions of Theorem 1.1 are satisfied (more precisely, in (1.7) the summand $\xi_{0} \lambda$ is replaced by $\left.\xi_{0} h(x, \lambda)\right)$.

\section{New genuine nonlinearity condition and the main result}

At the beginning of the section, we introduce a generalization of the nonlinearity condition (1.7), which we will use in the proof of Theorem 2.6.

Definition 2.1. We say that the vector $\left(f_{1}, f_{2}\right) \in\left(C^{1}\left(\mathbf{R} ; L^{\infty}\left(\mathbf{R}^{2}\right)\right)\right)^{2}$ satisfies generalized genuine nonlinearity conditions if there exists a function $h(x, y, \lambda) \in$ $C^{1}\left(\mathbf{R}_{\lambda} ; L^{\infty}\left(\mathbf{R}_{x}^{2}\right)\right)$ such that for a.e. $x \in \mathbf{R}^{2}$ and every $\xi \in S^{2}$ (sphere in $\mathbf{R}^{3}$ ),

$$
\begin{aligned}
\xi_{0} h(x, \lambda)+\xi_{1} f_{1}(x, \lambda)+\xi_{2} f_{2}(x, \lambda) \neq & \text { constant function in } \lambda \\
& \text { on any nontrivial interval. }
\end{aligned}
$$

In the following, we denote $\Pi=(0, \infty) \times \mathbf{R}^{2}=\mathbf{R}^{+} \times \mathbf{R}^{2}$. Furthermore, we denote by $W_{\mathrm{c}, \text { loc }}^{-1,2}(\Pi)$ families of functions that are precompact in $W_{\text {loc }}^{-1,2}(\Pi)$, and by $\mathcal{M}_{\mathrm{b}, \text { loc }}(\Pi)$ families of functions that are locally bounded in the space of Radon measures $\mathcal{M}(\Pi)$. 
We recall Murat's lemma.

Lemma $2.2([9])$. Assume that the family $\left(Q_{\varepsilon}\right)_{\varepsilon}$ is bounded in $L^{p}(\Omega), p>2$, $\Omega \subset \mathbf{R}^{d}$ is an open set. Then,

$$
\left(\operatorname{div} Q_{\varepsilon}\right)_{\varepsilon} \in W_{\mathrm{c}, \mathrm{loc}}^{-1,2}(\Omega) \text { if } \operatorname{div} Q_{\varepsilon}=p_{\varepsilon}+q_{\varepsilon}
$$

with $\left(q_{\varepsilon}\right)_{\varepsilon} \in W_{\mathrm{c}, \mathrm{loc}}^{-1,2}(\Omega)$ and $\left(p_{\varepsilon}\right)_{\varepsilon} \in \mathcal{M}_{\mathrm{b}, \mathrm{loc}}(\Omega)$.

In the following, we denote $u^{\varepsilon, \delta}$ to be the classical solutions to (1.8), (1.9). We will need the following a priory estimates (Lemmas $2.3-2.5$ ), essentially proved in [12].

LEMma 2.3. [12, Lemma 4.1] ( $L^{\infty}$-bound) There exists a constant $c_{1}>0$ such that for all $t>0$,

$$
\left\|u^{\varepsilon, \delta}(t, \cdot)\right\|_{L^{\infty}\left(\mathbf{R}^{2}\right)} \leq c_{1}
$$

LEMma 2.4. [12, Lemma 4.2] (Lipschitz regularity in time) If $\delta=c \varepsilon$, for a constant $c>0$, then there exists a constant $c_{2}$, independent of $\varepsilon$ and $\delta$, such that for all $t>0$,

$$
\iint_{\mathbf{R}^{2}}\left|u_{t}^{\varepsilon, \delta}(t, \cdot)\right| d x \leq c_{2} .
$$

LEMma 2.5. [12, Lemma 4.3] (Entropy dissipation bound) There exists a constant $c_{3}$, independent from $\varepsilon$ and $\delta$, such that for all $t>0$,

$$
\varepsilon \iint_{\mathbf{R}^{2}}\left(u_{x_{1}}^{\varepsilon, \delta}(t, \cdot)\right)^{2}+\left(u_{x_{2}}^{\varepsilon, \delta}(t, \cdot)\right)^{2} d x \leq c_{3} .
$$

Now, we can formulate the main theorem of the paper.

THEOREM 2.6. Assume that the flux function $\left(f_{1}, f_{2}\right)$ from (1.1) satisfy the generalized genuine nonlinearity conditions from Definition 2.1 and conditions (1.3)-(1.6). If $\varepsilon=c \delta$, then the family of solutions $\left(u^{\varepsilon}\right)_{\varepsilon} \equiv\left(u^{\varepsilon, \delta}\right)_{\varepsilon, \delta}$ to (1.8) is strongly precompact in $L_{\text {loc }}^{1}(\Pi)$.

Proof. In order to use (2.1), we rewrite (1.8) as

$$
\begin{aligned}
& \partial_{t} h\left(x, u^{\varepsilon, \delta}\right)+\partial_{x_{1}} f_{1}^{\delta}\left(x, u^{\varepsilon, \delta}\right)+\partial_{x_{2}} f_{2}^{\delta}\left(x, u^{\varepsilon, \delta}\right) \\
= & \partial_{t} h\left(x, u^{\varepsilon, \delta}\right)-u_{t}^{\varepsilon, \delta}+\varepsilon\left(u_{x_{1} x_{1}}^{\varepsilon, \delta}+u_{x_{2} x_{2}}^{\varepsilon, \delta}\right),
\end{aligned}
$$

where $x=\left(x_{1}, x_{2}\right) \in \mathbf{R}^{2}$. Denote $\eta^{\prime}(\lambda)=H(\lambda-k)$, for some constant $k$ (here $H$ stands for the Heaviside step function) and define the corresponding entropy fluxes:

$$
\begin{aligned}
q_{0}(x, \lambda) & =H(\lambda-k)(h(x, \lambda)-h(x, k)), \\
q_{i}(x, u, \lambda) & =H(\lambda-k)\left(f_{i}(x, \lambda)-f_{i}(x, k)\right), \quad i=1,2, \\
q_{i}^{\delta}(x, \lambda) & =H(\lambda-k)\left(f_{i}^{\delta}(x, \lambda)-f_{i}^{\delta}(x, k)\right), \quad i=1,2 .
\end{aligned}
$$


We multiply (2.2) by $\eta^{\prime}\left(u^{\varepsilon, \delta}\right)$ and add $\partial_{x_{1}} q_{1}\left(x, u^{\varepsilon, \delta}\right)$ and $\partial_{x_{2}} q_{2}\left(x, u^{\varepsilon, \delta}\right)$ on both sides of equality (2.2) to obtain

$$
\begin{aligned}
& \partial_{t} q_{0}\left(x, u^{\varepsilon, \delta}\right)+\partial_{x_{1}} q_{1}\left(x, u^{\varepsilon, \delta}\right)+\partial_{x_{2}} q_{2}\left(x, u^{\varepsilon, \delta}\right) \\
= & H\left(u^{\varepsilon, \delta}-k\right)\left(\partial_{t} h\left(x, u^{\varepsilon, \delta}\right)-D_{x_{1}} f_{1}^{\delta}(x, k)-D_{x_{2}} f_{2}^{\delta}(x, k)-u_{t}^{\varepsilon, \delta}\right) \\
& +\varepsilon\left(\partial_{x_{1}}\left(u_{x_{1}}^{\varepsilon, \delta} \eta^{\prime}\left(u^{\varepsilon, \delta}\right)\right)-\left(u_{x_{1}}^{\varepsilon, \delta}\right)^{2} \eta^{\prime \prime}\left(u^{\varepsilon, \delta}\right)+\partial_{x_{2}}\left(u_{x_{2}}^{\varepsilon, \delta} \eta^{\prime}\left(u^{\varepsilon, \delta}\right)\right)-\left(u_{x_{2}}^{\varepsilon, \delta}\right)^{2} \eta^{\prime \prime}\left(u^{\varepsilon, \delta}\right)\right) \\
& +\partial_{x_{1}}\left(q_{1}-q_{1}^{\delta}\right)\left(x, u^{\varepsilon, \delta}\right)+\partial_{x_{2}}\left(q_{2}-q_{2}^{\delta}\right)\left(x, u^{\varepsilon, \delta}\right) \\
\leq & H\left(u^{\varepsilon, \delta}-k\right)\left(\partial_{t} h\left(x, u^{\varepsilon, \delta}\right)-D_{x_{1}} f_{1}^{\delta}(x, k)-D_{x_{2}} f_{2}^{\delta}(x, k)-u_{t}^{\varepsilon, \delta}\right) \\
& +\varepsilon\left(\partial_{x_{1}}\left(u_{x_{1}}^{\varepsilon, \delta} \eta^{\prime}\left(u^{\varepsilon, \delta}\right)\right)+\partial_{x_{2}}\left(u_{x_{2}}^{\varepsilon, \delta} \eta^{\prime}\left(u^{\varepsilon, \delta}\right)\right)\right) \\
& +\partial_{x_{1}}\left(q_{1}-q_{1}^{\delta}\right)\left(x, u^{\varepsilon, \delta}\right)+\partial_{x_{2}}\left(q_{2}-q_{2}^{\delta}\right)\left(x, u^{\varepsilon, \delta}\right) \quad \text { in } \mathcal{D}^{\prime}(\Pi) .
\end{aligned}
$$

In order to use Theorem 1 we have to show that

$$
\operatorname{div}_{(t, x)}\left[\left(q_{0}, q_{1}, q_{2}\right)\left(x, u^{\varepsilon}\right)\right] \in W_{c, \text { loc }}^{-1,2}(\Pi) .
$$

From (2.3) and the Schwartz lemma on nonnegative distributions, it follows that there exists a Radon measure $\mu_{k}^{\varepsilon, \delta} \in \mathcal{M}(\Pi)$ such that

$$
\begin{aligned}
& \partial_{t} q_{0}\left(x, u^{\varepsilon}\right)+\partial_{x_{1}} q_{1}\left(x, u^{\varepsilon}\right)+\partial_{x_{2}} q_{2}\left(x, u^{\varepsilon}\right) \\
= & H\left(u^{\varepsilon}-k\right)\left(\partial_{t} h\left(x, u^{\varepsilon, \delta}\right)-D_{x_{1}} f_{1}^{\delta}(x, k)-D_{x_{2}} f_{2}^{\delta}(x, k)-u_{t}^{\varepsilon}\right) \\
& +\partial_{x_{1}}\left(q_{1}-q_{1}^{\delta}\right)\left(x, u^{\varepsilon}\right)+\partial_{x_{2}}\left(q_{2}-q_{2}^{\delta}\right)\left(x, u^{\varepsilon}\right) \\
& +\varepsilon\left(\partial_{x_{1}}\left(u_{x_{1}}^{\varepsilon} \eta^{\prime}\left(u^{\varepsilon}\right)\right)+\partial_{x_{2}}\left(u_{x_{2}}^{\varepsilon} \eta^{\prime}\left(u^{\varepsilon}\right)\right)\right)+\mu_{k}^{\varepsilon, \delta}(t, x) .
\end{aligned}
$$

Now, we use Lemma 2.2 to see that (2.4) holds. Indeed, from Lemma 2.4 we obtain that

$$
H\left(u^{\varepsilon}-k\right)\left(\partial_{\lambda} h\left(x, u^{\varepsilon}\right) u_{t}^{\varepsilon}-u_{t}^{\varepsilon}\right) \in \mathcal{M}_{\mathrm{b}, \mathrm{loc}}(\Pi) .
$$

Lemma 2.5 implies

$$
\partial_{x_{1}}\left(\varepsilon u_{x_{1}}^{\varepsilon} H\left(u^{\varepsilon}-k\right)\right)+\partial_{x_{2}}\left(\varepsilon u_{x_{2}}^{\varepsilon} H\left(u^{\varepsilon}-k\right)\right) \in W_{\mathrm{c}, \mathrm{loc}}^{-1,2}(\Pi),
$$

provided that

$$
\varepsilon u_{x_{1}}^{\varepsilon} H\left(u^{\varepsilon}-k\right) \rightarrow 0, \text { in } L_{\mathrm{loc}}^{2}(\Pi),
$$

and

$$
\int_{\Pi}\left|\varepsilon u_{x_{1}}^{\varepsilon} H\left(u^{\varepsilon}-k\right)\right|^{2} d x d t \leq \varepsilon^{2} \int_{\Pi}\left|u_{x_{1}}^{\varepsilon}\right|^{2} d x d t \leq T c \varepsilon \rightarrow 0, \quad \varepsilon \rightarrow 0 .
$$

Furthermore,

$$
\left.\left(D_{x_{1}} f_{1}^{\delta}(x, k)+D_{x_{2}} f_{2}^{\delta}(x, k)\right) H\left(u^{\varepsilon}-k\right)\right) \in \mathcal{M}_{\mathrm{b}, \text { loc }}(\Pi),
$$

since $f_{i}^{\delta} \in B V(\Pi)$. Finally,

$$
\partial_{x_{1}}\left(q_{1}-q_{1}^{\delta}\right), \partial_{x_{2}}\left(q_{2}-q_{2}^{\delta}\right) \in W_{\mathrm{c}, \mathrm{loc}}^{-1}(\Pi),
$$


since, according to (1.5),

$$
\begin{aligned}
\left|q_{i}-q_{i}^{\delta}\right| & \leq\left|f_{i}^{\delta}\left(x, u^{\varepsilon}\right)-f_{i}\left(x, u^{\varepsilon}\right)\right|+\left|f_{i}^{\delta}(x, k)-f_{i}(x, k)\right| \\
& \leq 2 \max _{a \leq p \leq b}\left|f_{i}^{\delta}(x, p)-f_{i}(x, p)\right| \rightarrow 0, \text { in } L_{\mathrm{loc}}^{2}\left(\mathbf{R}^{2}\right) .
\end{aligned}
$$

To prove that $\mu_{k}^{\varepsilon, \delta}$ belongs to $\mathcal{M}_{\mathrm{b}, \text { loc }}(\Pi)$, it is enough to prove that for any compact $K \subset \Pi$, it holds $\mu_{k}^{\varepsilon, \delta}(K)<c_{4}$, for a constant $c_{4}$ independent on $\varepsilon$ and $\delta$. To prove the latter, fix a function $\varphi_{K} \in C_{0}^{1}(\Pi)$ such that $\varphi_{K} \geq 0,\left|\nabla \varphi_{K}\right| \leq c_{5}$ for a constant $c_{5}>0$ independent on $\varepsilon, \delta$, and $\varphi_{K}(t, x)=1$ for $(t, x) \in K$. Then, since $\mu_{k}^{\varepsilon, \delta}$ are positive Radon measures for every $\delta, \varepsilon>0$, it follows from (2.5) that

$$
\mu_{k}^{\varepsilon, \delta}(K) \leq \int_{\Pi} \varphi_{K}(t, x) d \mu_{k}^{\varepsilon, \delta} \leq c_{4}\left|\nabla \varphi_{K}\right| \leq c_{6},
$$

where $c_{6}$ depends on the set $K$, the constants $c_{4}, c_{5}$, and the constants $c_{i}, i=1,2,3$, from Lemmas 2.3-2.5, but not on $\varepsilon, \delta>0$.

Collecting (2.5)-(2.10), from Murat's lemma we obtain (2.4). Applying Theorem 1.1 , we conclude the proof.

REMARK 2.1. Notice that Theorem 2.6 can be extended to the case when the dimension $d$ of the space is greater than two $(d>2)$ in a rather straightforward manner. More precisely, if we assume a multidimensional analogue of the generalized genuine nonlinearity condition, i.e., that there exists a function $h \in C^{1}\left(\mathbf{R} ; L^{\infty}\left(\mathbf{R}^{d}\right)\right)$ such that for a.e. $x \in \mathbf{R}^{d}$ and every $(\tau, \xi) \in S^{d}, \tau \in \mathbf{R}, \xi \in \mathbf{R}^{d}$ ( $S^{d}$ is the unit sphere in $\mathbf{R}^{d+1}$ ), the mapping (below, $\cdot$ denotes the scalar product)

$$
\begin{array}{r}
\lambda \mapsto \tau h(x, \lambda)+\xi \cdot f(x, \lambda) \text { is not a constant function in } \lambda \\
\text { on any nontrivial interval, }
\end{array}
$$

then the family $\left(u_{\varepsilon}\right)_{\varepsilon}$ of solutions to $(1.8),(1.9)$ is strongly precompact in $L_{\text {loc }}^{1}\left(\mathbf{R}^{+} \times\right.$ $\mathbf{R}^{d}$ ) for any $d \in \mathbf{N}$.

The latter remark is due to Professor E. Tadmor and an unknown referee. We express our deepest gratitude for this important observation.

\section{Examples}

In this section we give three examples which can not be dealt by using the known results.

a) First, we shall apply Theorem 2.6 on the following problem

$$
\begin{aligned}
u_{t}+(f(x, u))_{x} & =0, \\
\left.u\right|_{t=0}=u_{0}(x) & \in B V(\mathbf{R}), \quad a \leq u_{0}(x) \leq b,
\end{aligned}
$$

where a one-dimensional variant of (1.3)-(1.6) are satisfied. We assume that for almost every $x \in \mathbf{R}$ the mapping

$$
[a, b] \ni \lambda \mapsto f(x, \lambda),
$$

is different from a constant on any nontrivial interval.

Corollary 3.1. A family of solutions $\left(u^{\varepsilon}\right)_{\varepsilon}$ of the problem

$$
\begin{aligned}
u_{t}^{\varepsilon}+\left(f^{\varepsilon}\left(x, u^{\varepsilon}\right)\right)_{x} & =\varepsilon u_{x x}^{\varepsilon}, \\
\left.u^{\varepsilon}\right|_{t=0} & =u_{0}^{\varepsilon}(x), \quad(t, x) \in \mathbf{R}^{+} \times \mathbf{R}^{2},
\end{aligned}
$$


where the notation is taken from (1.8)-(1.9), is strongly precompact in $L_{\mathrm{loc}}^{1}\left(\mathbf{R}^{+} \times \mathbf{R}\right)$.

Proof. According to the previous theorem, it is enough to find a function $h(x, \lambda)$ such that the mapping

$$
\lambda \mapsto h(x, \lambda) \xi_{0}+f(x, \lambda) \xi_{1}
$$

is different from a constant on any nontrivial interval. Taking $h(x, \lambda)=f^{2}(x, \lambda)$, we conclude that (3.2) will not be satisfied only if there exists a nonzero set $\Omega \subset \mathbf{R}$ such that for $x \in \Omega$ there exists $\left(\xi_{0}, \xi_{1}\right) \in \mathbf{R}^{2} \backslash\{0\}$ satisfying

$$
f(x, \lambda)=\frac{-\xi_{1} \pm \sqrt{\xi_{1}^{2}+4 \xi_{0} c}}{2 \xi_{0}},
$$

for a constant $c$, contradicting (3.1).

b) Now, we consider the following example of a one dimensional conservation law with discontinuous flux,

$$
\begin{cases}\partial_{t} u+(H(x) f(u)+(1-H(x)) g(u))_{x}=0, & (t, x) \in \mathbf{R}^{+} \times \mathbf{R}, \\ \left.u\right|_{t=0}=u_{0}(x) \in B V(\mathbf{R}), \quad a \leq u_{0}(x) \leq b, & x \in \mathbf{R},\end{cases}
$$

where $f, g \in C^{1}(\mathbf{R}), f(a)=f(b)=g(a)=g(b)=0$, and $f$ and $g$ are not identically equal to zero on any non-degenerate subinterval of $(a, b)$. The problem has been thoroughly investigated in recent past (the following list is very incomplete $[1,2,3,4,5,7,11$, $13,14,15,17])$. In the following Corollary 3.2 , we shall prove that $\left(u^{\varepsilon}\right)_{\varepsilon}$ is strongly precompact in $L_{\mathrm{loc}}^{1}\left(\mathbf{R}^{+} \times \mathbf{R}\right)$ without any structural assumptions on the flux (such as genuine nonlinearity, crossing condition, convexity, a single crossing point of $f$ and $g$, etc.). This is a very important fact since an appropriate limit function along a subsequence is not only a weak solution to problem (3.3), but at the same time satisfies entropy admissibility conditions (see e.g. [14, Definition 1.2]). We remark that the existence of an entropy admissible weak solution to (3.3) is established in [5] also with no structural conditions on the flux.

Corollary 3.2. Let $\omega \in C_{0}^{1}(\mathbf{R})$ be a positive, compactly supported function with total mass one. Let $H_{\varepsilon}(z)=\int_{-\infty}^{z / \varepsilon} \omega\left(z^{\prime}\right) d z^{\prime}$ represents a regularization of the Heaviside function. A family of solutions $\left(u^{\varepsilon}\right)_{\varepsilon}$ of the problem

$$
\begin{aligned}
u_{t}^{\varepsilon}+\partial_{x}\left(H_{\varepsilon}(x) f(u)+\left(1-H_{\varepsilon}(x)\right) g(u)\right)_{x} & =\varepsilon u_{x x}^{\varepsilon}, \\
\left.u^{\varepsilon}\right|_{t=0} & =u_{0}^{\varepsilon}(x),
\end{aligned}
$$

is strongly precompact in $L_{\mathrm{loc}}^{1}\left(\mathbf{R}^{+} \times \mathbf{R}\right)$.

Proof. If

$$
\lambda \mapsto f(\lambda), \quad \lambda \mapsto g(\lambda),
$$

are non-constant on any interval $(\alpha, \beta) \subset(a, b)$ then we can apply Corollary 3.1.

Otherwise, we take disjoint intervals $\left(\alpha_{i}^{f}, \beta_{i}^{f}\right) \subset(a, b), i=1, \ldots, d_{1}, d_{1} \in \mathbf{N} \cup\{\infty\}$, where the function $f$ is constant, and the disjoint intervals $\left(\alpha_{i}^{g}, \beta_{i}^{g}\right) \subset(a, b), i=1, \ldots, d_{2}$, 
$d_{2} \in \mathbf{N} \cup\{\infty\}$, where the function $g$ is constant. Then, we take the functions $\hat{f}, \hat{g} \in$ $\operatorname{Lip}(\mathbf{R})$ :

$$
\begin{aligned}
& \hat{f}(\lambda)=\left\{\begin{array}{ll}
0, & \lambda \notin\left(\alpha_{i}^{f}, \beta_{i}^{f}\right), \\
\left(\lambda-\alpha_{i}^{f}\right)\left(\lambda-\beta_{i}^{f}\right), & \lambda \in\left(\alpha_{i}^{f}, \beta_{i}^{f}\right),
\end{array} \quad i=1, \ldots, d_{1},\right. \\
& \hat{g}(\lambda)= \begin{cases}0, & \lambda \notin\left(\alpha_{i}^{g}, \beta_{i}^{g}\right), \quad i=1, \ldots, d_{2} . \\
\left(\lambda-\alpha_{i}^{g}\right)\left(\lambda-\beta_{i}^{g}\right), & \lambda \in\left(\alpha_{i}^{g}, \beta_{i}^{g}\right),\end{cases}
\end{aligned}
$$

Now, the vector $\left(H(x)\left(f^{2}+\hat{f}\right)(u)+(1-H(x))\left(g^{2}+\hat{g}\right)(u), H(x) f(u)+(1-H(x)) g(u)\right)$ is genuinely nonlinear, implying that $(u, H(x) f(u)+(1-H(x)) g(u))$ satisfies the generalized genuine nonlinearity condition. Thus, we can apply Theorem 2.6 to complete the proof of the corollary.

c) Consider the following Cauchy problem:

$$
\begin{aligned}
& u_{t}+(k(x) g(u))_{x}+(l(y) f(u))_{y}=0 \\
& \left.u\right|_{t=0}=u_{0}(x, y) \in B V\left(\mathbf{R}^{2}\right)
\end{aligned}
$$

with $-1 \leq u_{0}(x, y) \leq 1$, and

$$
g(u)= \begin{cases}0, & \text { for }|u| \geq 1 \\ u+1, & \text { for }-1<u \leq 0, \quad k(x)= \begin{cases}3, & \text { for } x \geq 0 \\ 1, & \text { for } x<0\end{cases} \\ 1-u^{2}, & \text { for } 0<u<1\end{cases}
$$

and

$$
f(u)= \begin{cases}0, & \text { for }|u| \geq 1 \\
1-u^{2}, & \text { for }-1<u \leq 0, \quad l(y)=\left\{\begin{array}{ll}
4, & \text { for } y \geq 0 \\
1-u, & \text { for } 0<u<1
\end{array} 2 \text { for } y<0,\right.\end{cases}
$$

The flux vector $(k(x) g(u), l(x) f(u))$ does not satisfy the classical genuine nonlinearity condition and we can not apply results from [12]. Indeed, for any $x \in(-1,0)$, $y \in \mathbf{R}$ (and the set $(0,1) \times \mathbf{R}$ has infinite measure), choose $\left(\xi_{0},-\xi_{0}, 0\right) \in S^{2}$. Mapping (1.7) becomes

$$
\lambda \mapsto \xi_{0} \lambda-\xi_{0}(\lambda+1)=-\xi_{0},
$$

and this is constant in $\lambda$.

Therefore, to state that a weak solution to (3.5) exists, we have to use generalized genuine nonlinearity condition (2.1). According to Theorem 2.6 , the family $\left(u^{\varepsilon}\right)_{\varepsilon}$ of solutions to the equation

$$
u_{t}^{\varepsilon}+\left(k_{\varepsilon}(x) g\left(u^{\varepsilon}\right)\right)_{x}+\left(l_{\varepsilon}(y) f\left(u_{\varepsilon}\right)\right)_{y}=\varepsilon\left(u_{x x}^{\varepsilon}+u_{y y}^{\varepsilon}\right)
$$

where

$$
k_{\varepsilon}(x)=\left\{\begin{array}{ll}
3, & \text { for } x \geq \varepsilon \\
\frac{x}{\varepsilon}+2, & \text { for }-\varepsilon<x<\varepsilon \\
1, & \text { for } x \leq-\varepsilon,
\end{array} \quad \text { and } \quad l_{\varepsilon}(y)= \begin{cases}4, & \text { for } x \geq \varepsilon \\
\frac{x}{\varepsilon}+3, & \text { for }-\varepsilon<x<\varepsilon \\
2, & \text { for } x \leq-\varepsilon,\end{cases}\right.
$$


is strongly precompact in $L_{\text {loc }}^{1}\left(\mathbf{R}^{+} \times \mathbf{R}^{2}\right)$. Indeed, take $h$ from $(2.1)$ to be $h(x, u)=u^{3}$. In that case, the vector field $(h(x, u), k(x) g(u), l(y) f(u))$ satisfies conditions from Theorem 2.6, since $k, l \in B V$. Therefore, Theorem 2.6 provides strong $L_{\text {loc }}^{1}$-precompactness of the family $\left(u^{\varepsilon}\right)_{\varepsilon}$. Clearly, a strong $L_{\text {loc }}^{1}$-limit along a subsequence of $\left(u^{\varepsilon}\right)_{\varepsilon}$ will represent a weak solution to (3.5).

\section{REFERENCES}

[1] Adimurthi, G.D.V. Gowda, Conservation law with discontinuous flux, J. Math. Kyoto Univ., $43,1,27-70,2003$.

[2] Adimurthi, S. Mishra and G.D.V. Gowda, Existence and stability of entropy solutions for a conservation law with discontinuous non-convex fluxes, Netw. Heterog. Media, 2(1), 127 157 (electronic), 2007.

[3] E. Audusse and B. Perthame, Uniqueness for scalar conservation laws with discontinuous flux via adapted entropies, Proc. Roy. Soc. Edinburgh Sect. A, 135(2), 253-265, 2005.

[4] F. Bachmann and J. Vovelle, Existence and uniqueness of entropy solution of scalar conservation laws with a flux function involving discontinuous coefficients, Commun. Part. Differ. Equs., 31(1-3), 371-395, 2006.

[5] Bürger, K.H. Karlsen and J. Towers, An Engquist-Osher-type scheme for conservation laws with discontinuous flux adapted to flux connections, SIAM J. Numer. Anal., 47(3), 1684$1712,2009$.

[6] C.M. Dafermos, Hyperbolic Conservation Laws in Continuum Physics, Grundlehren der Mathematischen Wissenschaften, [Fundamental Principles of Mathematical Sciences], SpringerVerlag, Berlin, 325, 2000.

[7] S. Diehl, A uniqueness condition for non-linear convection-diffusion equations with discontinuous coefficients, J. Hyperbolic Differ. Equs., to appear.

[8] R.J. DiPerna, Measure-valued solutions to conservation laws, Arch. Rational Mech. Anal., 88(3), 223-270, 1985.

[9] L.C. Evans, Weak Convergence Methods in Nonlinear Partial Differential Equations, AMS, Providence, Rhode Island, 74, 1990.

[10] P. Gerard, Microlocal defect measures, Commun. Part. Differ. Equs., 16(11), 1761-1794, 1991.

[11] E.F. Kaasschieter, Solving the Buckley-Leverett equation with gravity in a heterogeneous porous medium, Comput. Geosci., 3(1), 23-48, 1999.

[12] K.H. Karlsen, M. Rascle and E. Tadmor, On the existence and compactness of a twodimensional resonant system of conservation laws, Commun. Math. Sci., 5(2), 253-265, 2007.

[13] K.H. Karlsen, N.H. Risebro and J.D. Towers, On a nonlinear degenerate parabolic transportdiffusion equation with a discontinuous coefficient, Electron. J. Differ. Eqs., 93(23), (electronic) 2002.

[14] K.H. Karlsen and N.H. Risebro and J.D. Towers, $L^{1}$ stability for entropy solutions of nonlinear degenerate parabolic convection-diffusion equations with discontinuous coefficients, Skr. K. Nor. Vidensk. Selsk., 3, 1-49, 2003.

[15] K.H. Karlsen and J.D. Towers, Convergence of the Lax-Friedrichs scheme and stability for conservation laws with a discontinous space-time dependent flux, Chinese Ann. Math. Ser. B, 25(3), 287-318, 2004.

[16] S.N. Kruzhkov, First order quasilinear equations in several independent variables, Mat. Sb., 81, 1309-1351, 1970.

[17] D. Mitrovic, New entropy conditions for the scalar conservation law with discontinuous flux, preprint, http://www.math.ntnu.no/conservation/2009/013.html

[18] Panov and E. Yu., Existence and strong precompactness properties for entropy solutions of a first-order quasilinear equation with discontinuous flux, Arch. Rational Mech. Anal., doi 10.1007/s00205-009-0217-x.

[19] M. Rascle and D. Serre, Compacite par compensation et systemes hyperboliques de lois de conservation, Applications C.R. Acad. Sci. Paris Ser. I Math., 299(14), 673676, 1984.

[20] L. Tartar, Compensated compactness and applications to partial differential equations, Nonlinear analysis and mechanics: Heriot-Watt Symposium, Res. Notes in Math., Pitman, Boston, Mass.-London, Vol. IV, 39, 136-212, 1979.

[21] L. Tartar, H-measures, a new approach for studying homogenisation, oscillations and concentration effects in partial differential equations, Proc. Roy. Soc. Edinburgh Sect. A, 115, 3-4, 193-230, 1990. 